\title{
9 Simulation of the Early Stages of Growth on a Patterned Substrate
}

\author{
L. Nurminen, A. Kuronen, and K. Kaski \\ Helsinki University of Technology, Laboratory of Computational Engineering, \\ P.O.Box 9400, 02015 HUT, Finland
}

\begin{abstract}
Kinetic Monte Carlo simulations are used to investigate nucleation of two-dimensional islands on a substrate with nanoscale patterning. The patterned substrate is incorporated into a basic solid-on-solid model by introducing inhomogeneous energy barriers for surface diffusion. Growth on a patterned substrate promotes the spatial ordering of islands, which also leads to a narrow island size distribution. The optimal growth conditions are determined by the length scale of the substrate pattern.
\end{abstract}

\subsection{Introduction}

Much of recent interest in the studies of metallic and semiconductor systems has been devoted to epitaxially grown nanostructures due to their great potential for technological applications [1]. For example, spontaneous selforganization of nanoscale islands in heteroepitaxial thin-film growth has been utilized to manufacture semiconductor quantum dots. Before this method can be used to develop actual devices, the growth must be controllable at a microscopic level.

Here we investigate the possibility of growing ordered nanostructure arrays by using substrates with periodically arranged patterns. Our work is motivated by recent experiments on the system of $\mathrm{Ag} / 2 \mathrm{ML} \mathrm{Ag} / \mathrm{Pt}(111)[2,3]$. The equilibrium structure of the second monolayer of $\mathrm{Ag}$ on $\mathrm{Pt}(111)$ is a network of crossing dislocations that form in order to relieve lattice-mismatch induced strain. Due to their mutual repulsion, the dislocations arrange into ordered periodic patterns which can be transferred into nanostructure superlattices upon deposition of further material. It was suggested in [2] that the dislocations act repulsively towards diffusing adatoms, which explains the observed confinement of nucleated islands.

\subsection{Kinetic Monte Carlo Simulations}

The KMC method is applied to investigate the time evolution of the growing surface. The simulations are based on a solid-on-solid (SOS) bond-counting model [4] that gives a good qualitative description of MBE (molecular beam epitaxy) growth. New material is deposited onto an initially flat surface at 
constant rate $F$ expressed in monolayers per second (ML/s). Desorption under usual MBE conditions is negligible, thus it is omitted from the model, and the fractional surface coverage is given by $\Theta=F t$. The diffusion rate of a single adatom is given by the Arrhenius-type activation based expression

$$
k(E, T)=k_{0} \exp \left(-E / k_{\mathrm{B}} T\right),
$$

where the prefactor is assigned the value $k_{0}=2 k_{\mathrm{B}} T / h\left(k_{\mathrm{B}}\right.$ is the Boltzmann constant and $h$ is the Planck constant). The activation energy is given by $E=E_{\mathrm{S}}+n E_{\mathrm{N}}$ where $E_{\mathrm{S}}$ stands for substrate binding energy and $E_{\mathrm{N}}$ describes the contribution of binding to lateral nearest neighbors of the initial site. $n=0-4$ is the number of occupied nearest neighbors.

The patterned substrate was incorporated into the basic diffusion model by introducing periodic variation into the diffusion activation energies. For this purpose, the lattice was divided into square-shaped domains of size $l \times l$. The square geometry was adopted for simplicity and to speed up the computation. The diffusion barriers were varied within these domains using two different models:

1. Model $A$. The parameter $E_{\mathrm{S}}$ was let to vary piecewise linearly as a function of the adatom lateral position on the surface. The total diffusion barrier is given by $E_{\mathrm{A}}=E_{\mathrm{S}}\left(x_{i}, y_{i}\right)+n E_{\mathrm{N}}$ where the index $i$ refers to the initial site.

2. Model B. An additional barrier $E_{\mathrm{D}}$ was introduced for jumps directed toward domain boundaries. The strength of the additional barrier is determined by the distance of the adatom from the boundary. The total barrier for diffusion from site $i$ to a neighboring site $j$ is given by $E_{\mathrm{B}}=E_{\mathrm{S}}+n E_{\mathrm{N}}+E_{\mathrm{D}}(i, j)$.

Note that in model A the hopping rate is determined by the lateral position of the adatom on the surface, but from a given lattice site $i$ the adatom hops with equal probability to any of the four neighboring sites. In the case of model $\mathrm{B}$, the diffusion barrier depends on both the initial and the final site of the diffusion jump.

The simulations were carried out on a square lattice of size $L \times L$ with periodic boundaries. The domain size was selected to be $22 \times 22$. The following parameter values were used: $E_{\mathrm{S}}=0.75 \mathrm{eV}$ (variation in model A $\pm 0.10 \mathrm{eV}$ ), $E_{\mathrm{N}}=0.18 \mathrm{eV}, E_{\mathrm{D}, \max }=0.02 \mathrm{eV}$ (next to boundaries), $E_{\mathrm{D}, \min }=0 \mathrm{eV}$ (at domain centers), $F=0.0033 \mathrm{ML} / \mathrm{s}$, and $T=340-440 \mathrm{~K}$. Ensemble averages were calculated from 50 simulation runs for each temperature.

\subsection{Results}

Figure $9.1 \mathrm{a}-\mathrm{b}$ shows examples of island morphologies for islands grown on a homogeneous and a patterned substrate, respectively. We see that the islands 

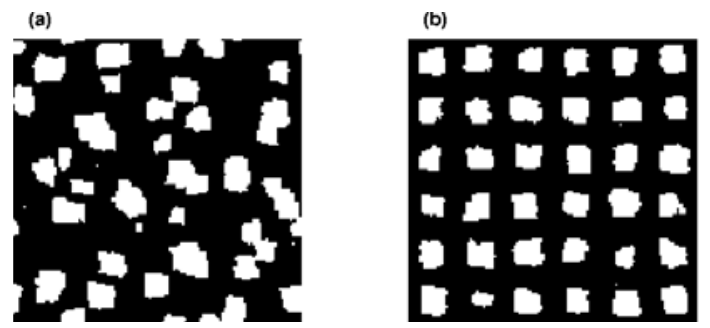

Fig. 9.1. Snapshots of KMC simulations showing growing islands (a) on a homogeneous substrate and (b) on a patterned substrate. The temperature is $390 \mathrm{~K}$ and the coverage is $15 \% .132 \times 132$ surface sections are displayed. Dark areas designate the substrate and light areas the first layer of adatoms

on the patterned substrate order into a regular arrangement which displays the periodicity of the underlying pattern. This confinement effect depends strongly on the growth temperature that determines the average diffusion length of the adatoms. The samples shown in Fig. 9.1 were obtained at $T=$ $390 \mathrm{~K}$. At this temperature, the average diffusion length for the given set of model parameters is of the same order of magnitude as the lateral length $l$ of the domains. This means that on average the adatoms are able to visit the whole unit cell before they join an existing island or nucleate a new one. Due to the inhomogeneous diffusion barriers, the net flow of adatoms is directed toward the domain centers, thus increasing the nucleation probability in these areas. The combined result of these two effects is that within a certain temperature range exactly one island nucleates in each unit cell of the substrate structure.

Figure $9.2 \mathrm{a}$ shows the island size distributions at $T=390 \mathrm{~K}$ for random and ordered nucleation on a homogeneous and patterned substrate, respectively. The size distributions are normalized according to scaling theory [5]. We see that the spatial ordering leads to an enhanced size uniformity of the islands. The domains act as equally large capture areas for the islands, since all material deposited into a certain domain stays confined within this domain due to the biased diffusion. This holds for temperatures at which the average diffusion length corresponds to the length scale of the substrate pattern.

The temperature dependencies of the total island densities, $N_{x}$, are shown in Fig. 9.2b. In the patterned substrate case, the island density remains approximately constant for temperatures in the range $380-400 \mathrm{~K}$. This constant density corresponds to the case where each domain contains a single island. At lower temperatures, the island density increases, which means that several small islands nucleate within each domain due to the shorter diffusion length. At high temperatures, diffusion across the domain boundaries becomes activated. Some of the domains are left empty as the adatoms join islands in surrounding domains, thus decreasing the island density. 
(a)

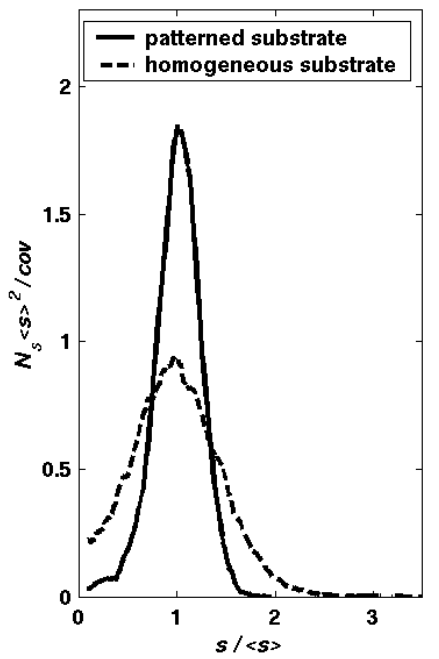

(b)

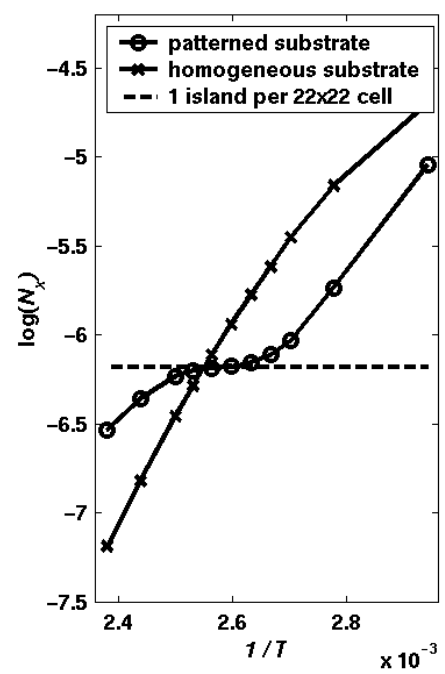

Fig. 9.2. (a) Scaled island size distributions for random nucleation and ordered nucleation. The coverage is $15 \%$. The model parameters correspond to $D / F \approx 10^{6}$. The distributions are normalized according to scaling theory ( $s$ denotes the island size in atoms, $\langle s\rangle$ its average value, and $N_{s}$ is the areal density of islands composed of $s$ atoms). (b) Arrhenius plot of island densities $N_{x}$ for nucleation on a homogeneous and a patterned substrate

So far we have not distinguished between the two patterned substrate models. The results discussed above are for model A. We did not include model B here, because the results are very similar (details can be found in [6]). Differences between models A and B become apparent when the total diffusion rate of an adatom in different positions on the surface is considered. In the case of model $\mathrm{A}$, the adatoms are more tightly bound to the substrate the closer they are to the domain centers. Thus diffusion is fast near the boundaries and slow near the centers of the domains. The energetically most favorable configuration is a single island located at the center of each domain. In the case of model B, the hop-direction dependence of the diffusion barriers produces a net movement of adatoms toward the domain centers, but the change in the total diffusion rate is very small $(0.02 \mathrm{eV}$ at maximum compared to the maximum variation of $0.2 \mathrm{eV}$ for model A). Under favorable growth conditions, the islands order into a regular array, but post-deposition relaxation of the structure leads to the dissociation of small islands in favor of larger ones (see [6] for details). This Ostwald ripening process does not occur in model A. 


\subsection{Conclusions}

In summary, we have employed kinetic Monte Carlo simulations on a microscopic scale to study the nucleation process of two-dimensional islands on a patterned substrate. Our results show that periodic inhomogeneity in the diffusion activation energies significantly affect the early stages of growth. Under favorable conditions, growth on a patterned substrate leads to the formation of an ordered array of islands. The length scale of the substrate pattern determines the optimal growth temperature (or equivalently the optimal particle flux at a fixed temperature). The results of our simulations are in good qualitative agreement with experimental observations of Brune and coworkers [2].

\section{Acknowledgments}

We would like to thank Professor David P. Landau for a number of helpful discussions. This work was supported by the Academy of Finland, project on Computational Research of Semiconductor Materials, Project No. 1169043 (Finnish Center of Excellence Program 2000-2005).

\section{References}

1. D. Bimberg, M. Grundmann, N. Ledentsov: Quantum Dot Hterostructures, (Wiley, Chichester, 1999)

2. H. Brune, M. Giovannini, K. Bromann, K. Kern: Nature 394, 451 (1998)

3. K. Bromann, M. Giovannini, H. Brune, K. Kern: Eur. Phys. J. D 9, 25-28 (1999)

4. A.-L. Barabási, H. E. Stanley: Fractal Concepts in Surface Growth, (Cambridge University Press, 1995)

5. H. Brune: Surf. Sci. Rep. 31, 121 (1998)

6. L. Nurminen, A. Kuronen, K. Kaski: Phys. Rev. B 63, 035407 (2001) 